\title{
How To Interpret Elevated Plasmatic Level of High-Sensitive Troponin $T$ in Newborns and Infants?
}

\author{
P. JEHLICKA ${ }^{1}$, M. HUML ${ }^{1}$, D. RAJDL ${ }^{3}$, A. MOCKOVA ${ }^{2}$, M. MATAS $^{2}$, J. DORT $^{2}$, \\ A. MASOPUSTOVA ${ }^{1}$
}

${ }^{1}$ Department of Pediatrics, Faculty of Medicine in Pilsen, Charles University in Prague, Faculty Hospital in Pilsen, Czech Republic, ${ }^{2}$ Department of Neonatology, Faculty of Medicine in Pilsen, Charles University in Prague, Faculty Hospital in Pilsen, Czech Republic, ${ }^{3}$ Department of Clinical Hematology and Clinical Chemistry, Faculty of Medicine in Pilsen, Charles University in Prague, Faculty Hospital in Pilsen, Czech Republic

Received July 3, 2017

Accepted November 22, 2017

On-line January 5, 2018

\section{Summary}

Research and clinical implications on novel cardiac biomarkers has intensified significantly in the past few years. The highsensitive troponin $\mathrm{T}$ (hscTnT) assay plays a dominant role in diagnostic algorithm regarding myocardial injury in adults. Despite generally accepted use of hscTnT there are no data about physiological concentrations and cut-off limits in neonates and infants to date. The aim of this study is to assess hscTnT levels in healthy newborns and infants. Consecutively 454 healthy full termed newborns and 40 healthy infants were enrolled in the study. Samples of cord or venous blood were drawn and tested for hscTnT concentrations with high-sensitive TnT assay (Roche Cobas e602 immunochemical analyzer). The 97.5 percentile of hscTnT concentration was assessed and correlation analysis was performed in neonates. Two hundred and thirteen samples (47\%) were excluded due to blood hemolysis of various degrees in neonates. Finally, the group of 241 healthy newborns was statistically analyzed. The median concentration of hscTnT was $38.2 \mathrm{ng} / \mathrm{ml}$, 97.5 percentile reached $83.0 \mathrm{ng} / \mathrm{l}$ (confidential interval 74.1 to $106.9 \mathrm{ng} / \mathrm{l}$ ). HscTnT concentrations were statistically decreased in hemolytic samples when compared to non-hemolytic samples $(34.3 \mathrm{ng} / \mathrm{l}$ [26.7 to $42.0 \mathrm{ng} / \mathrm{l}]$ and $37.1 \mathrm{ng} / \mathrm{l}$ [30.5 to $47.9 \mathrm{ng} / \mathrm{l}]$, respectively, $\mathrm{p}=0.003$ ). Elevated plasma concentrations of hscTnT decreased to adult level within six months. This study has confirmed the higher reference levels of hscTnT in neonates and young infants when compared with adult population. Many extracardiac factors as hemolysis and age may affect the hscTnT level. Based on presented results, a careful clinical interpretation of hscTnT is recommended.

\section{Key words}

High sensitive troponin T • Infants • Myocardial damage • Newborns

\section{Corresponding author}

M. Huml, Department of Pediatrics, Faculty of Medicine in Pilsen, Faculty Hospital, Charles University in Prague, Alej Svobody 80, 30400 Pilsen, Czech Republic. Fax: +420 377104694. E-mail: humlm@fnplzen.cz

\section{Introduction}

Cardiac troponins are sensitive markers of myocardial damage and are also powerful prognostic indicators of adverse cardiac events. With advances in technology, a new era in troponin assays has started. The ability of the high-sensitive troponin $\mathrm{T}$ (hscTnT) assay to detect troponin $\mathrm{T}$ elevation in blood within two hours and with higher sensitivity after a myocardial insult led to the replacement of former troponin assays. However, conventional cardiac troponin $\mathrm{T}$ and I concentrations might be elevated in newborns despite a normal cardiovascular state (Adamcová et al. 1995). To date, there is limited information on high-sensitive troponin I in children (Caselli et al. 2016). However, there are no reliable data regarding hscTnT reference levels in newborns and infants. The primary aim of this study was to determine the hscTnT distribution in healthy neonates. The secondary objective 
was to correlate hscTnT with birth weight, blood gases, degree of hemolysis in neonates and to assess changes of hscTnT concentrations during early infancy.

\section{Methods}

This study was approved by the local Ethics Committee and performed according to the principles of the Declaration of Helsinki. After written informed consent was obtained from legal guardians, 454 full-term healthy newborns were enrolled in the study. All subjects were recruited from the single neonatology department at the university hospital. Exclusion criteria included an Apgar score less than 8 points performed at standard intervals of time after birth, the presence of congenital defects or infection, and a history of chronic medication or drug abuse in the mother.

Samples of cord blood were drawn in the tube with lithium heparin as a blood anticoagulant and tested for hscTnT concentrations and blood gases. The electrochemiluminescent (ECLIA) method was used to assess the hscTnT concentration (Roche Cobas e602 immunochemical analyzer). The level of hemolysis was measured by a photometrical Cobas 8000 analyzer. Samples with hemolysis higher than $1 \mathrm{~g} / \mathrm{l}$ (hemolysis index $\geq 2$ ) were excluded from further analysis, respecting the recommendations of the analytical manufacturer. Blood gases and acid-base balance analysis were performed using a GEM 3500 analyzer according to the standardized methodology of the university hospital.

HscTnT concentrations were not normally distributed and therefore the medians and interquartile ranges are reported and non-parametric comparisons were made. We constructed a reference range of cardiac troponin $\mathrm{T}$ concentrations and calculated the upper limit in the population $\left(97.5^{\text {th }}\right.$ percentile). Differences among groups were examined by the Mann-Whitney test. Correlations among hscTnT and other parameters (birth weight, level of hemolysis, umbilical cord acid base status) were analyzed using the rank correlation coefficient. A p-value of 0.05 or less was considered statistically significant.

Table 1. Demographic data.

\begin{tabular}{lccc}
\hline & Girls & Boys & p-value \\
\hline Probands & 114 & 127 & \\
Gestational age at birth (weeks) & $39.0(39 ; 40)$ & $39.0(39 ; 40)$ & NS \\
Birth weight $(\mathrm{kg})$ & $3.310(3.030 ; 3.600)$ & $3.510(3.207 ; 3.837)$ & 0.001 \\
Apgar score $\left(1^{1 t}\right.$, $^{\text {th }}$ and $10^{\text {th }}$ min) & $10(10 ; 10), 10(10 ; 10), 10(10 ; 10)$ & $10(10 ; 10), 10(10 ; 10), 10(10 ; 10)$ & NS \\
Umbilical cord blood acid-base analysis at delivery & & \\
pH & $7.33(7.29 ; 7.33)$ & $7.34(7.30 ; 7.38)$ & NS \\
Base excess & $-2.7(-5.1 ;-1.1)$ & $-3.3(-5.6 ;-1.3)$ & NS \\
hscTnT $(n g / l)$ & $36.7(29.2 ; 47.1)$ & $38.7(33.0 ; 52.8)$ & 0.05 \\
hscTnT $(n g / l)-97.5^{\text {th }}$ percentile & 71.55 & 93.67 & \\
Hemolysis index & $0(0 ; 1)$ & $0(0 ; 1)$ & NS \\
\hline
\end{tabular}

The values are expressed as median and inter-quartile range $\left(25^{\text {th }}-75^{\text {th }}\right.$ percentile $)$ in the brackets; hscTnT, high-sensitivity troponin T; hemolysis index, scale from 0-5: grade $0(0-0.5 \mathrm{~g} / \mathrm{l})$, grade $1(0.51-1.0 \mathrm{~g} / \mathrm{l})$, grade $2(1.01-2.0 \mathrm{~g} / \mathrm{l})$, grade $3(2.01-3.0 \mathrm{~g} / \mathrm{l})$, grade 4 $(3.01-4.0 \mathrm{~g} / \mathrm{l})$, grade $5(4.01 \mathrm{~g} / \mathrm{l}$ and more).

\section{Results}

Cord blood samples were taken from 454 healthy newborns; 213 samples were excluded due to a blood hemolysis index $\geq 2$. The subset of 241 samples was statistically analyzed (Table 1). The median hscTnT concentration was $38.2 \mathrm{ng} / 1$ [interquartile range 31.3 to $48.0 \mathrm{ng} / \mathrm{l}]$. The distribution of hscTnT values in the study group is shown in Figure 1. The lower and upper limits of the hscTnT reference range were calculated as $20.1 \mathrm{ng} / 1$ (95\% CI 15.3 to $21.8 \mathrm{ng} / \mathrm{l})$ and $83.0 \mathrm{ng} / 1$ (95\% CI, 74.1 to $106.9 \mathrm{ng} / \mathrm{l})$, respectively. The difference between hscTnT concentrations in boys and girls tended to be statistically significant $(38.7 \mathrm{ng} / 1$ [33.0 to $52.8 \mathrm{ng} / 1]$ and $36.7 \mathrm{ng} / 1$ [29.2 to $47.1 \mathrm{ng} / \mathrm{l}]$, respectively, $\mathrm{p}=0.052$ ). The concentration of hscTnT was found to be significantly 
lower in newborns delivered via Cesarean section when compared with vaginal delivery $(35.0 \mathrm{ng} / \mathrm{l}$ [28.5 to $43.4 \mathrm{ng} / 1$ ] and $38.9 \mathrm{ng} / 1$ [32.6 to $48.4 \mathrm{ng} / 1$ ], respectively, $\mathrm{p}=0.0084$ ) (Fig. 2). HscTnT values were significantly lower in hemolytic blood samples (hemolysis index $\geq 3$ ) when compared with non-hemolytic samples $(34.3 \mathrm{ng} / \mathrm{l}$ [26.7 to $42.0 \mathrm{ng} / \mathrm{l}$ ] and $37.1 \mathrm{ng} / 1$ [30.5 to $47.9 \mathrm{ng} / \mathrm{l}$, respectively, $\mathrm{p}=0.003)$. The hscTnT level correlated inversely with the base status $(\mathrm{BE}$ : $r h o=-0.14, \mathrm{p}=0.03$ ) and with the $\mathrm{pH}$ level of the umbilical cord blood (rho=-0.14, $\mathrm{p}=0.03$ ). Elevated plasma concentrations of hscTnT decreased to adult range within six months (Fig. 3).

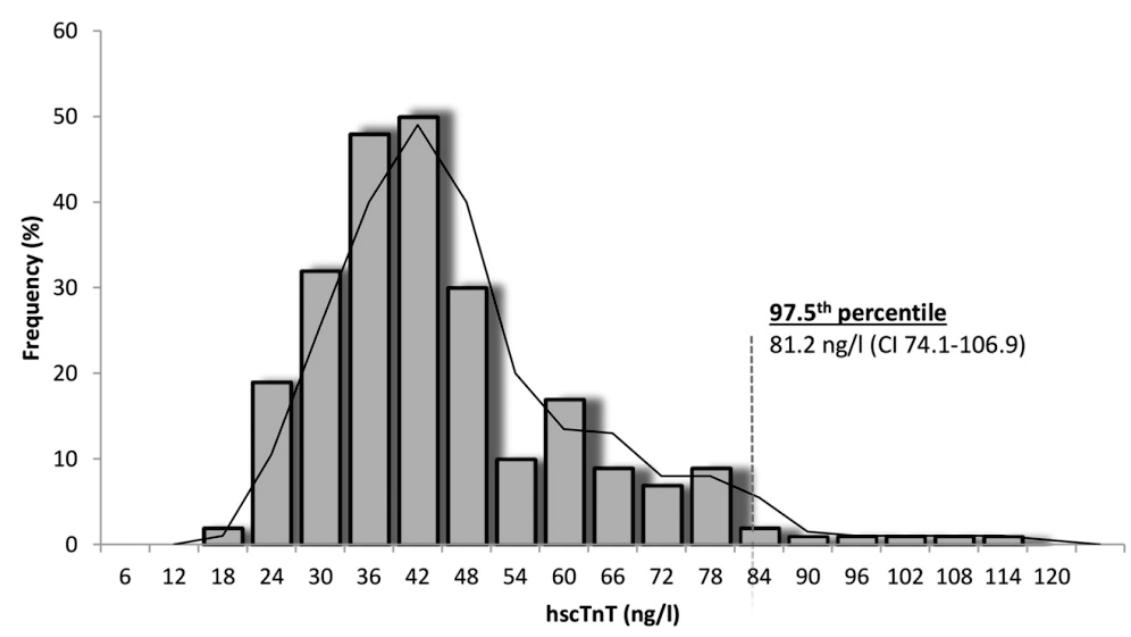

Fig. 1. Distribution of hscTnT values in healthy newborns $(n=241)$. hscTnT, high-sensitive troponin $\mathrm{T}$; $\mathrm{CI}$, confidential interval.

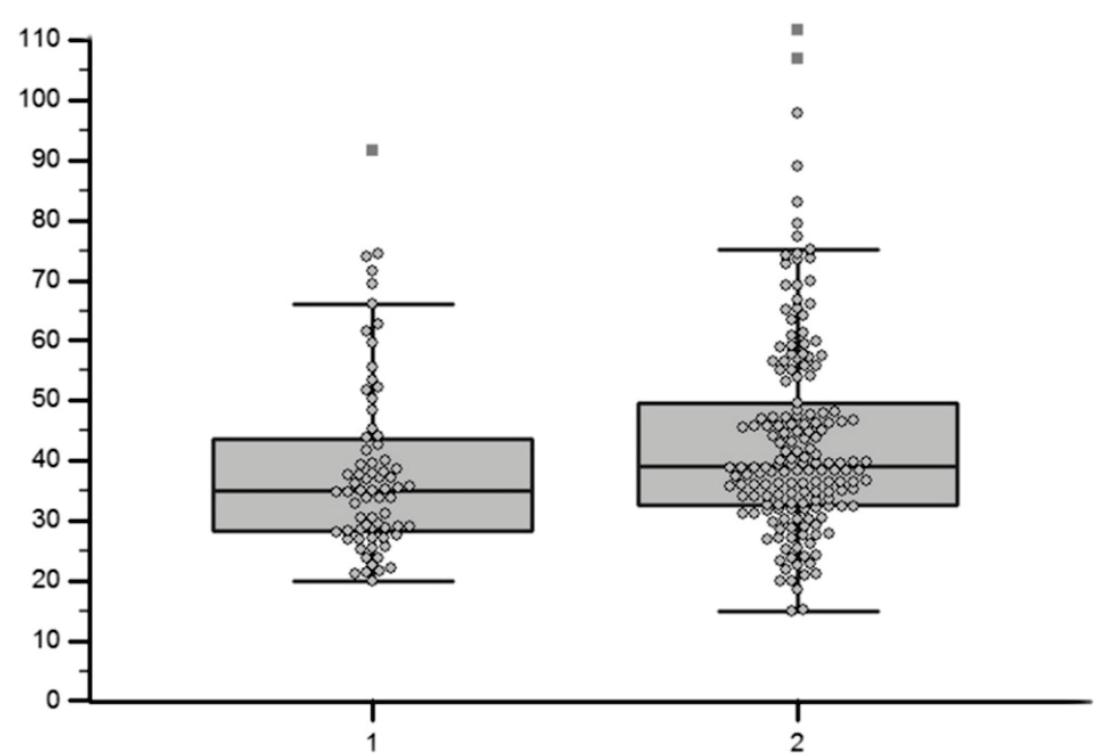

Fig. 2. hscTnT concentration values according to the delivery method. Boxes indicate the inter-quartile range. Horizontal lines within boxes indicate medians. Whiskers extend to the highest or lowest value. 1. Cesarean section $(n=70) 2$. Vaginal delivery $(n=171)$.

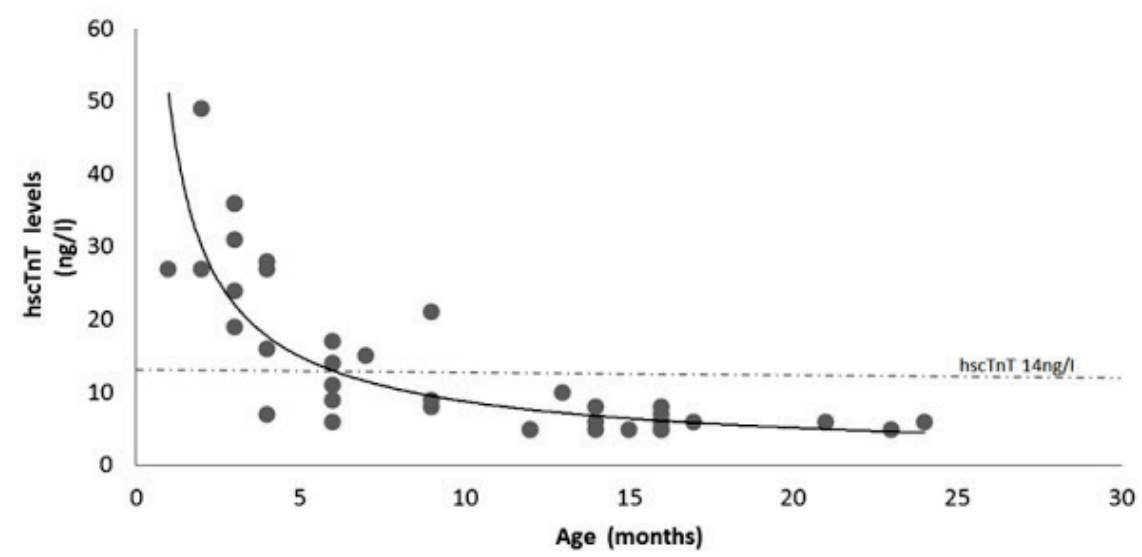

Fig. 3. Relationship of the hscTnT levels to the age of probands. 


\section{Discussion}

Our study presents the reference range of hscTnT concentrations calculated for healthy newborns. The upper limit of hscTnT was significantly higher when compared with adult population $(83.0 \mathrm{ng} / \mathrm{l}$ and $13.5 \mathrm{ng} / \mathrm{l}$, respectively) (Giannitsis et al. 2010). This finding is in agreement with studies using conventional troponin $\mathrm{T}$ and I assays in healthy neonates (Baum et al. 2004, Clark et al. 2001). The reason for this elevation remains questionable. The influence of physical stress and transient hypoxia during vaginal birth could not be excluded (Costa et al. 2007, Cruz et al. 2006). This theory is supported by studies reporting an elevation of cardiac troponin $\mathrm{T}$ in healthy adults after strenuous physical exertion (Apple 2009, Hewing et al. 2015). Indeed, hscTnT values were significantly higher after vaginal birth when compared to surgical delivery in our study (Fig. 2). However, neither physical stress nor transient perinatal hypoxia fully elucidates the elevated concentrations of hscTnT in healthy newborns and young infants. It is well known that elevated plasma concentrations of TnT decline to normal levels in successfully treated adults within two weeks (Skeik et al. 2008). In our study, elevated plasma levels of hscTnT in healthy neonates and infants return to adult physiological range substantially slower. This process persisted up to six months (Fig. 3). When excluded extracardiac pathological causes such as sepsis, renal failure, severe anemia or hyperbilirubinemia, another hypothesis might be taken into account. Initiation of spontaneous breathing promotes changes in pulmonary circulatory system in newborns. The decreasing pulmonary arterial resistance leads to reduction of the right ventricle afterload and the dominance is shifted from the right to the left ventricle within first months of life. We speculate that this physiological process linked with subtle structural myocardial changes might release a small amount of troponins into the circulation. These are detectable by high-sensitive TnT assay during newborn period and early infancy.

Hemolysis is known to negatively interfere in hscTnT assays. Up to $45 \%$ of our samples contained some degree of hemolysis. This result is in agreement with a former study ( $\mathrm{Li}$ et al. 2013) and it is of critical importance when interpreting hscTnT concentration. A hemoglobin concentration of $1.9 \mathrm{~g} / 1$ or higher might decrease the hscTnT level up to $20 \%$. We found a significant reduction in the hscTnT concentration at hemoglobin concentrations $\geq 2.0 \mathrm{~g} / 1$ (hemolysis index $\geq 3$ ). Consideration of preanalytical variables is essential, such as time interval from blood sampling to laboratory analysis. A significant decline in the hscTnT concentration over time has been reported ( $\mathrm{Li}$ et al. 2013). The acceleration in hemolysis might be explained by the release of erythrocyte proteases, causing a disruption in the TnT-epitope used in the diagnostic kit.

Higher hscTnT concentrations in boys when compared to girls in our study are in contrast to results of previous study using the conventional troponin $\mathrm{T}$ assay (Baum et al. 2004). However, this small difference in hscTnT concentrations has no clinical relevance.

\section{Strengths and limitations}

Our study was conducted on a quite large number of neonates. Despite excluding up to $45 \%$ of samples due to hemolysis (hemolysis index $\geq 2$ ), a distribution curve was constructed with sufficient statistical power. On the other hand, probands were not followed-up after study. A longitudinal study would require repeated blood sampling in healthy infants which is ethically questionable.

\section{Conclusion}

Substantially higher reference plasma concentrations of hscTnT in neonates and young infants when compared with the adult population require careful clinical interpretation. It is advised to consider many extracardiac factors as hemolysis and age that might affect the hscTnT levels in these children.

\section{Conflict of Interest}

Elecsys Troponin I Ths STAT lab sets were provided by ROCHE without any financial ties to the investigators.

\section{Acknowledgements}

The authors thank ROCHE for the provision of the reagents for the hscTnT assessment. This study was conducted within the research project PROGRES O-39.

\section{References}

ADAMCOVÁ M, KOKSTEIN Z, PALICKA V, PODHOLOVÁ M, KOSTÁL M: Troponin T levels in the cord blood of healthy term neonates. Physiol Res 44: 99-104, 1995. 
APPLE FS: A new season for cardiac troponin assays: it's time to keep a scorecard. Clin Chem 55: 1303-1306, 2009.

BAUM H, HINZE A, BARTES P, NEUMAIER D: Reference values for cardiac troponins T and I in healthy neonates. Clin Biochem 37: 1079-1082, 2004.

CASElli C, CANGEMI G, MASOTTI S, RAGUSTA R, GENNAI I, DEL RY S, PRONTERA C, CLERICO A: Plasma cardiac troponin I concentrations in healthy neonates, children and adolescents measured with a high sensitive immunoassay method: high sensitive troponin I in pediatric age. Clin Chim Acta 458: 68-71, 2016.

CLARK SJ, NEWLAND P, YOXALL CW, SUBHEDAR N: Cardiac troponin T in cord blood. Arch Dis Child Fetal Neonatal Ed 84: 34-37, 2001.

COSTA S, ZECCA E, De ROSA G, DE LUCA D, BARBATO G, PARDEO M, ROMAGNOLI C: Is serum troponin T a useful marker of myocardial damage in newborn infants with perinatal asphyxia? Acta Paediatr 96: 181-184, 2007.

CRUZ MA, BREMMER YA, PORTER BO, GULLQUIST SD, WATTERBERG KL, ROZYCKI HJ: Troponin T and cardiac dysfunction in extremely low-birth-weight infants. Pediatr Cardiol 27: 396-401, 2006.

GIANNITSIS E, KURZ K, HALLERMAYER K, JARAUSCH J, JAFFE AS, KATUS HA: Analytical validation of a high-sensitivity cardiac troponin T assay. Clin Chem 56: 254-261, 2010.

HEWING B, SCHATTKE S, SPETHMANN S, SANAD W, SCHROECKH S, SCHIMKE I, HALLECK F, PETERS H, BRECHTEL L, LOCK J, BAUMANN G, DREGER H, BORGES AC, KNEBEL F: Cardiac and renal function in a large cohort of amateur marathon runners. Cardiovasc Ultrasound 13: 13, 2015.

LI A, BRATTSAND G: Stability of serum samples and hemolysis interference on the high neonates with pathological jaundice. Laboratory Medicine 28: 267-270, 2013.

SKEIK N, PATEL DC: A review of troponins in ischemic heart disease and other conditions. Int J Angio 16: 53-58, 2007. 